\title{
LIDERAZGO, MANAGEMENT, ÉTICA EMPRESARIAL \\ Y LOS PRINCIPIOS DEL AIKIDO \\ UN ENFOQUE AUSTRIACO \\ PARA LA SUPERACIÓN DE LOS \\ MODELOS ÉTICOS ERRÓNEOS
}

CÉSAR MARTÍNEZ MESEGUER ${ }^{1}$

Fecha de recepción: 10 de noviembre de 2015

Fecha de aceptación: 14 de febrero de 2016

\section{I \\ INTRODUCCIÓN}

El principal objetivo de este trabajo es el de resaltar las importantes diferencias existentes entre los valores que se pretenden transmitir a través de la práctica del deporte competitivo - y más en concreto la práctica de artes marciales-, y aquellas otras que se transmiten a través del aikido, un arte marcial que trasciende y supera los aspectos negativos que perviven y forman parte del deporte y de las artes marciales de combate. Siguiendo esa misma argumentación, queremos demostrar y difundir que muchos de los valores que se pretenden transmitir tomando como ejemplo la competición deportiva, ya no tienen sentido en una sociedad evolucionada. Resultando mucho más adecuados aquellos principios planteados por el aikido, para la formación de las personas en general y, por lo

${ }^{1}$ Doctor en Derecho, profesor de la asignatura de Economía y Evolución del Master de Economía Austriaca de la Universidad Rey Juan Carlos de Madrid y de la asignatura de Introducción a la Economía en el Instituto de Estudios Bursátiles. Cinturón negro de aikido y de kárate. Blog: martinezmeseguerblog.wordpress.com. Correo: cesar.m.meseguer@hotmail.com 
tanto, para la elaboración de los contenidos de los cursos de liderazgo y de Management en particular, que se generen en el futuro, ya que, hasta el momento presente una gran parte de los principios, tácticas e, incluso, vocabulario utilizado para la formación de políticos, directivos de empresa, etc., se han derivado en su mayoría de las artes de la guerra y marciales, así como de los deportes competitivos que, en muchos casos, tienen un origen o fundamento en las anteriores, ya que aparecieron como formas de entrenamiento de guerreros y militares.

Las ideas que en concreto nosotros consideramos superadas son todas aquellas que, directa o indirectamente, están relacionadas con cualquier tipo de agresividad, violencia o lucha de contrarios. Aspectos estos que tenían sentido en las épocas en las que surgieron, pero que en la actualidad deben ser totalmente apartados de la educación y de la formación. Los valores de una sociedad más primitiva, guerrera y basada en el triunfo del que resulte vencedor en la lucha, deben quedar atrás, si lo que se pretende es alcanzar una sociedad más abierta y libre, en la que se generen principios que permitan la colaboración (por encima de la imposición), el trabajo conjunto y basado en acuerdos, así como la posibilidad de que todos salgan beneficiados sin que existan ni vencedores ni venci$\mathrm{dos}^{2}$, todo ello dentro de ese complejo entramado de relaciones

2 Según la moderna teoría económica subjetivista y, fundamentalmente, a partir de las aportaciones de Carl Menger (uno de los precursores de la denominada Revolución marginalista - junto con Jevons y Walras-, y fundador de la Escuela Austriaca de Economía) resulta obvio que para que se produzca un intercambio libre en el mercado, las valoraciones de los individuos que intercambian — que por definición son subjetivas- respecto de los bienes que van a ser intercambiados, implican que las mismas deben ser opuestas y distintas, en el sentido de que lo que yo quiero entregar debe tener menos valor para mí que lo que quiero adquirir, y viceversa respecto del otro sujeto que intercambie. De no ser así, el intercambio no se llevaría a cabo. Esto significa que, salvo que se produzca un error empresarial, todo intercambio de bienes y/o servicios en el mercado libre genera beneficios para todos los que intercambian, lo que unido a la idea de que el mercado (entendido en su concepción dinámica) genera continuos procesos de creación de riqueza — con bienes y servicios nuevos, mejores, más abundantes, etc. - y que dicha riqueza no es, por tanto, una especie de «tarta» dada que hay que repartir (que sería la forma errónea en la que la concepción estática entiende el mercado), permite formular la idea de que bajo unos principios adecuados de comportamiento, todos los agentes del mercado ganen en los procesos de interrelación, incluso los que no participan de un intercambio en concreto, sólo por el mero 
sociales que son el mercado y la propia sociedad, tal y como se defiende en el seno de la Escuela Austriaca de Economía.

No debe olvidarse que, aunque todas las artes marciales podrían integrarse dentro de lo que en japonés se conoce como budo ${ }^{3}$, éste, a su vez, tal y como advertía Tamura Nabuyoshi", puede interpretarse con sentidos diferentes, según las personas que lo definan, ya que lo que para unos es «fuerza destructiva», para otros es «fuerza de paz», y es precisamente esta última opción la elegida por el aikido, que, al implicar un máximo respeto a la vida (la propia y la del otro), respeta por igual la integridad física y mental de uno mismo y la del compañero, desapareciendo la idea de rivalidad y de contrario y, por lo tanto, de choque de fuerzas - predominante en el resto de artes marciales-, para ser sustituida por la idea de unificación y reconducción de flujos de energía ${ }^{5}$.

Lamentablemente, una gran mayoría de los deportistas y entrenadores tienen como objetivo principal, y casi me atrevería a decir que único, el ganar a toda costa, llevándoles esta mentalidad incluso a cometer todo tipo de trampas, a recurrir al dopaje y a la utilización de sustancias prohibidas, etc. Actitud que se aprecia más todavía en el mundo profesional, donde esto se ha convertido en

hecho de formar parte de esa sociedad, generándose cada vez más riqueza, siendo falsa la afirmación de que para que unos ganen otros deban necesariamente perder.

${ }^{3}$ El budo es un término japonés que se refiere al conocimiento técnico de las artes marciales japonesas y al conjunto de normas éticas que sus practicantes deben respetar. La traducción de dicho termino vendría a ser algo así como «el camino, o la vía, marcial»; pero con la idea implícita de que el objetivo último es la obtención de la paz a través de la formación marcial, por lo que trasmite también la idea de que, tanto la paz interior como la exterior, se pueden alcanzar a través de la aplicación de determinadas disciplinas marciales, situando la importancia de dicha vía, más en el lado espiritual y meditativo de sus enseñanzas, haciendo hincapié en la búsqueda de la perfección de uno mismo, lo que tendrá su reflejo en el exterior. Esta forma de pensar no es exclusiva del $b u d o$, y es perfectamente trasladable a otras ceremonias y artes japoneses.

${ }^{4}$ Tamura Nabuyoshi (1933-2010) fue Shihan, $8^{\circ}$ dan de aikido otorgado por Aikikai Hombu Dojo, y uno de los principales discípulos del creador del aikido, Morihei Ueshiba. Fue, además, autor de varios libros dedicados a la enseñanza de este arte marcial, siendo pieza clave en la gran difusión que el mismo tiene hoy día en Francia.

${ }^{5}$ Como muy bien analiza en sus clases Yasunari Kitaura, Shihan, $8^{\circ}$ dan de aikido otorgado por Aikikai Hombu Dojo. Kitaura es licenciado en Historia del arte por la Universidad Waseda de Tokio y se doctoró por la Universidad Complutense de Madrid, por lo que, además de ser un gran maestro de aikido, es un gran conocedor de la cultura occidental, en general, y de la española, en particular. 
una auténtica lacra, ya por desgracia muy generalizada. Antiguamente, cuando saltaba un escándalo de dopaje parecía una aberración, hoy en día son tantos los escándalos en los que se destapan auténticas redes mafiosas dentro del deporte (futbol, atletismo, ciclismo, natación, boxeo, etc., etc., etc.) que, salvo contadas excepciones, parece que se trata de actos que tenemos tristemente asumidos. El reflejo de todo lo que venimos comentando, se aprecia, no sólo en el deporte, sino también en toda la sociedad, donde los escándalos a nivel empresarial y de corrupción política son incontables, generándose muchas veces de forma conjunta, al verse involucrados políticos, federaciones deportivas, empresarios y patrocinadores, y los propios deportistas, evidentemente.

Es cierto que, por desgracia, ningún ámbito de la vida se ve libre de la acción de malas personas que buscan cualquier resquicio para saltarse las reglas y sacar beneficio a costa de los demás, pero también es verdad que determinados principios mal escogidos y determinadas actitudes erróneas, fomentan la posibilidad de que algunos aspectos no deseados y acciones negativas proliferen sin control; o bien al contrario, es decir, el hecho de que la selección de determinados principios correctos fomenta el desarrollo de aspectos y acciones que se consideran beneficiosos para facilitar la vida en sociedad, quedando marginados los anteriores. De esta manera, el beneficio social a largo plazo de optar por la vía más adecuada es enorme y difícilmente calculable ${ }^{6}$. Está pues en nuestras manos la elección y la clase de mundo que queremos vivir y dejar en herencia a las futuras generaciones. ¿Qué tipo de dirigentes y líderes queremos para nuestra sociedad? ¿Qué tipo de ética y de principios queremos que les guíen?

La filosofía pacífica basada en la cooperación y la no agresión, justa y honesta del aikido, sólo pretende aportar su pequeño grano de arena allí donde se aplique, y uno de esos ámbitos debería ser precisamente el que ha motivado este trabajo: la importantísima

${ }^{6}$ La relevancia de este complejo proceso social de selección de normas morales y éticas, ha sido analizado por el autor del presente trabajo en su obra titulada La Teoría evolutiva de las instituciones, reseñada en la bibliografía citada al final del texto, basándose en los estudios previos de dos de los principales autores de la Escuela Austriaca de Economía, F. A. Hayek y C. Menger. 
labor que los líderes y empresarios (entendidos en sentido amplio: profesionales, autónomos, administradores de sociedades, consejeros, directores, etc.) cumplen en la sociedad con sus acciones y su ejemplo.

Para empezar, y esto puede resultar sorprendente para las personas que no conozcan este arte marcial, en el aikido no hay combate, ni competiciones, ni vencedores ni vencidos, todos ganan. En el aikido hay, de esta manera, una mutua colaboración entre tori y $u k e^{7}$, siendo el único objetivo que mueve a ambos, el disfrute de la propia actividad y la mejora personal a todos los niveles. Y decimos a todos los niveles con conocimiento de causa, ya que, a nivel físico, proporciona equilibrio, coordinación, agilidad, elasticidad, velocidad de reacción, etc., mientras que, a nivel mental y psicológico, proporciona autocontrol, equilibrio emocional, rectitud, generosidad, cortesía, honestidad, respeto, etc. Aspectos todos ellos que trascienden y se trasladan a los ámbitos colectivos y a la propia sociedad ${ }^{8}$.

${ }^{7}$ El término tori (sinónimo de shite y de nage) se refiere en japonés al que realiza una técnica ante un oponente que le ataca, el cual recibe el nombre de uke o aite, desempeñando un papel de agresor. Aunque, como ya hemos mencionado, no es un verdadero adversario, ya que es un compañero que ataca de una forma predeterminada e indicada por el sensei, para colaborar en el aprendizaje del otro compañero. Seguidamente, tori y uke intercambian los papeles. En un entrenamiento normal las parejas de tori y uke van rotando para que, al final, todos hayan entrenado con todos, aunque suelen colocarse alumnos con cinturones de grado superior con los que están comenzando, para facilitar la corrección de los posibles errores de los principiantes.

8 A este respecto es muy importante destacar la importancia de seleccionar adecuadamente al maestro (sensei) que queremos que nos enseñe, es fundamental coincidir con sus planteamientos y con la forma de impartir sus clases, si no queremos desilusionarnos rápidamente. Debe tenerse en cuenta que cada maestro tiene una forma distinta de enseñar: unos hacen más hincapié en el aspecto técnico, otros en el físico, otros dan más relevancia a los aspectos espirituales o a la tradición. Unos aplican las técnicas con mucha suavidad mientras que otros lo hacen de forma más contundente. Con ello queremos decir que no es que sean mejores o peores, sino que debemos buscar al que tenga una forma de enseñar que encaje mejor con nuestra manera de ser y con lo que estamos buscando, para que la práctica del aikido sea verdaderamente gratificante y placentera. 


\section{II LUCHA VS. COLABORACIÓN}

El mundo empresarial siempre se ha caracterizado, a lo largo de la historia, por estar basado en la idea de lucha contra los competidores, con el único objetivo final consistente en que sólo alcanzara el triunfo aquél que se consideraba más fuerte en el mercado; a costa, incluso, de la destrucción de los demás en el proceso descrito, lo que llevaba a considerar que, en cualquier caso, la posición de monopolista era la que resultaba más beneficiosa para una empresa. Hoy día sabemos que esta teoría está basada en una mala interpretación de la teoría darwinista que, de una forma simplista y errónea, ve la evolución, única y exclusivamente, como una lucha que debe culminar con el triunfo del más fuerte (en el sentido de mejor dotado física y/o mentalmente) ${ }^{9}$. Esta idea, que afortunadamente está siendo totalmente superada ${ }^{10}$ gracias, entre otras, a las propias aportaciones efectuadas por la Escuela Austríaca en este campo, hizo, tal y como hemos adelantado en la introducción, que durante mucho tiempo se aplicaran en los cursos de liderazgo y en los de Management, principios extraídos de lo que se ha venido en denominar «el arte de la guerra»" ${ }^{11}$, en todas sus diferentes versiones: bushido tradicional japonés -inspirado fundamentalmente en los estrictos códigos samuráis- o en diferentes artes marciales orientales (judo, kárate, taekwondo...), etc. La idea esencial de todas estas teorías era siempre una consecuencia lógica de lo anterior: el triunfo frente al contrario, la lu-

\footnotetext{
${ }^{9}$ Cuando, en realidad, a nivel humano estaríamos hablando del triunfo del que mejor se adapte y supere las adversidades, y no hay mejor forma de hacerlo que con el aumento de nuestra capacidad de resiliencia, unido a la colaboración con los demás, para poder superar cualquier obstáculo. Lo que se sitúa muy por encima, evidentemente, de la debilidad espiritual y mental, y de la idea de lucha y confrontación.

${ }^{10}$ A este respecto, F. A. Hayek advirtió además de las diferencias existentes entre la evolución biológica y la evolución social, así como respecto de la relevancia que en la última de ellas tienen los conceptos de colaboración y de transmisión de información, tal y como se aprecia en su obra La fatal arrogancia, obra reseñada en la bibliografía del presente trabajo.

${ }^{11}$ Recordemos el libro clásico del mismo título El Arte de la Guerra, de Sun Tzu.
} 
cha o la guerra como principio último... «matar para no morir» ${ }^{12}$, etc.

Por suerte, esta forma de ver el mercado y la dirección empresarial está variando totalmente gracias a que las más serias y recientes investigaciones en distintos campos del saber y en diferentes ciencias naturales y sociales, están llegando a la conclusión de que los verdaderos procesos de evolución son extremadamente más complejos que la interpretación excesivamente simplista que se estaba haciendo del darwinismo hasta hace muy poco tiempo. Las relaciones entre los seres vivos y su entorno nos demuestran que lo que verdaderamente conduce al progreso y la superación de las dificultades, no es una lucha encarnizada y violenta donde sólo el que aparenta ser, en un momento dado, el más apto triunfa, sino que, en realidad, las fuerzas que verdaderamente nos hacen evolucionar en una línea ascendente, son unos principios bien distintos a los que se venían planteando y que en resumidas cuentas podrían englobarse en dos:

a) La autosuperación ${ }^{13}$ (frente a la superación del supuesto contrario).

b) La colaboración mutuamente enriquecedora (frente a la idea de lucha).

Esta forma de pensar plantea, además, la importante idea de que en una sociedad ${ }^{14}$ evolucionada, rica, próspera y que se desarrolla en

${ }^{12}$ El propio Adam Smith escribió en su obra Investigación sobre la naturaleza y causa de la riqueza de las naciones (La riqueza de las naciones) que «el arte de la guerra es ciertamente la más noble de todas las artes», por lo que, evidentemente, sus principios debían ser imitados.

${ }^{13}$ Con este concepto de autosuperación no nos referimos a la simple idea de «mejorar mis marcas» típicas del mundo del deporte (atletismo, natación...), sino a la idea de mejora como persona en todos los sentidos.

${ }^{14}$ Entendiendo sociedad en su sentido más amplio, que incluye lo que denominamos mercado, ya que, en última instancia, la sociedad es una complejísima red de interrelaciones humanas de intercambio, dentro de las cuales se situarían los intercambios que tienen una plasmación en precios monetarios, como una categoría mínima y particular. A este respecto resulta muy interesante el análisis efectuado por el profesor Huerta de Soto en su obra Socialismo, cálculo económico y función empresarial, reseñada en la bibliografía de este trabajo 
paz, el menos afortunado o el que fracasa, siempre tendrá abierta la posibilidad de superarse y, lo que resulta más llamativo y revolucionario de este planteamiento, vivirá de una forma infinitamente superior que el que lo hace en una sociedad basada en las ideas contrarias. Argumento éste ampliamente defendido por los autores de la Escuela Austriaca, al atacar cualquier tipo de sociedad totalitaria e intervencionista que coarte la libertad de los individuos. Por ello, este razonamiento va mucho más allá de lo que parece a simple vista, ya que correctamente elaborado, nos demuestra que incluso el que se considera vencedor en un mundo en «perpetua guerra», siempre vivirá peor y sus «triunfos» serán mucho menores que el de aquél que pueda considerarse menos favorecido de entre los que tengan la suerte de vivir en un mundo próspero y en paz. Según este planteamiento, el cacique de la tribu guerrera, el señor feudal o el samurai, siempre vivirían peor que si su actividad se hubiese desarrollado en un contexto evolutivo libre, donde los principios fuesen la autosuperación, tal y como la hemos definido, y la colaboración enriquecedora con los demás.

III

\section{ESTUDIOS QUE AVALAN LA TESIS SOSTENIDA EN EL PRESENTE TRABAJO}

Curiosamente, estas ideas que, tal y como hemos indicado, están siendo avaladas y que se sustentan en los estudios de diferentes disciplinas, y que tienen unos fundamentos científicos y lógicos muy sólidos, siguen sin introducirse plenamente en los distintos campos estudiados del liderazgo político, de la Administración y Dirección de Empresas, en los MBA, etc., o en todo lo referente a la cultura y la ética empresariales; ámbitos en los que el vocabulario típicamente bélico y, por tanto, las ideas de competición con el resto, de lucha, de triunfo frente al contrario, etc. siguen teniendo mucha fuerza.

Por otra parte, recientes estudios sobre Psicología del Deporte ${ }^{15}$ vienen confirmando las afirmaciones que mantenemos en el presen-

${ }^{15}$ A este respecto en el presente trabajo hemos tomado como referencia el contenido (así como la bibliografía citada) del estudio publicado en la Revista de Psicología 
te trabajo, respecto de que los beneficios del aikido en sus practicantes son mucho mayores que los que proporcionan el deporte en general y cualquier arte marcial de contacto con base competitiva. Aunque en muchos aspectos los estudios sobre los beneficios de la práctica de artes marciales son contradictorios en el sentido de que aprecian tanto puntos positivos como negativos, al referirse estos a la práctica del aikido en concreto y a las enseñanzas que de él se desprenden, se comprueba como los aspectos negativos observados en otras artes marciales prácticamente desaparecen, viéndose reforzados todos los aspectos positivos, añadiéndose otros nuevos que refuerzan nuestra postura respecto del salto cuantitativo y cualitativo que el aikido supone.

Algunos de los estudios realizados han evidenciado un mayor grado de agresividad entre los practicantes de boxeo que entre los que practicaban judo, kárate o aikido. Mientras que, de entre estos últimos, los aikidokas han demostrado tener una mayor motivación por el disfrute de la propia experiencia del aprendizaje, por la mejora personal y por el dominio de la actividad desempeñada, mostrando un mayor respeto por los principios éticos en los que se fundamenta, y a los que ya nos hemos referido, así como por el oponente, etc., frente al resto de artistas marciales (y deportistas en general), que tenían una mayor propensión hacia el ego, a competir comparándose con los demás y a tener como principal motivación la recompensa externa. Es decir, los practicantes de artes marciales de contacto pleno y competitivo son más propensos a comparar sus logros con los de los demás, a intentar ganar con el menor esfuerzo posible, e incluso a creer que el «éxito» se consigue si se usan trampas o engaños ${ }^{16}$, etc.

Es cierto que los adultos de cierta edad y experiencia tienden a sentir una mayor atracción por el aikido que los más jóvenes, quizá

del Deporte titulado «Factores motivacionales relacionados con la agresividad en las artes marciales», cuyas autoras son Lucia Monacis, Valeria de Pablo y Maria Sinatra, de las Universidades de Foggia, Verona y Bari (Italia), respectivamente, y que fue publicado en el Volumen 24, $n^{\circ} 1$, pp. 163-169 de dicha revista.

${ }_{16}$ Tal y como puede apreciarse, entre otros estudios, en el realizado por Gernigon, C. y Le Bras, H. (2000), « Achievement goals in aikido and judo: a comparative study among beginner and experienced practitioners». Journal of Applied Sport Psychology, 12(2), 168-179. 
porque con la edad uno se va acostumbrando a comportarse según determinados patrones morales y éticos, mientras que se prioriza la propia independencia y el simple placer de la práctica por encima de la competición. Pero también es cierto que son, precisamente, los beneficios de la práctica del aikido los que pueden ser una poderosa arma de formación para los jóvenes, en el sentido de que facilita el aprendizaje y el respeto a unos principios éticos esenciales, a disfrutar del placer de realizar una actividad en sí misma con ilusión, sin esperar siempre gratificaciones externas. Si el motivo principal de la acción es ganar trofeos o quedar primero en un ranking, dicha actitud es fácil que termine por degenerar en que al deportista sólo le interese competir para obtener recompensas y premios, dando preponderancia al hecho de vencer a toda costa, lo que finalmente lleva, de una forma paradójica e inevitable, a desarrollar comportamientos no «deportivos»y antisociales. De ahí el importante instrumento educativo que es el aikido, ya que enseñando a superar la necesidad psicológica de competencia y de derrota del contrario como único fin, se fomenta un carácter más maduro e independiente, que facilita la convivencia social, al estar más orientado al respeto de las normas éticas que facilitan la vida en sociedad, aumentando el respeto por los demás —a los que ya no se ve como simples oponentes y escollos en el camino, sino como iguales y colaboradores-. De tal manera que, utilizando el aikido como medio, se aprendería que lo que verdaderamente es importante es la percepción que tenemos de nosotros mismos y la auto-mejora que nos lleva a depurar nuestras competencias, conocimientos y valores, lo que nos permitiría disfrutar más de lo que hacemos, aumentando el respeto hacia los demás y hacia nosotros mismos, reduciendo la exaltación de nuestro ego y disminuyendo la agresividad.

La obsesión por vencer a toda costa para tener beneficios y obtener reconocimiento social, termina por generar comportamientos inmorales consistentes en la violación de las reglas de juego, aumentando la agresividad y el desprecio por los otros (y estamos hartos de ver este tipo de actitudes a nivel deportivo, empresarial y político). Sin embargo, no se hace nada por solucionar este grave problema, se sigue hablando de lo importante que es el respeto a los principios éticos esenciales y el respeto a los demás, pero segui- 
mos formando a nuestros jóvenes, que serán los líderes del futuro, en la idea de que lo importante es vencer al contrario a toda costa (el fin justifica los medios), acabar con la competencia, ganar dinero como sea y obtener reconocimiento y poder social. Lo que genera una sociedad «esquizofrénica», en el sentido de que se sabe lo que es bueno y, sin embargo, se fomenta todo lo contrario, como si se hubiera perdido la auténtica noción de la realidad.

Por estos motivos, nosotros consideramos que las importantes oportunidades que nos ofrece el aikido para tener una formación adecuada y positiva, no deben desperdiciarse, ya que correctamente utilizadas pueden dar unos frutos muy interesantes a todos los niveles.

\section{IV \\ EL AIKIDO \\ UN GRAN DESCONOCIDO}

Llegados a este punto es importante destacar que, curiosamente, fue dentro de las propias artes marciales donde se llegó a la misma conclusión que aquí estamos exponiendo. Y fue precisamente un artista marcial japonés llamado Morihei Uesiba ${ }^{17}$, el que fue capaz de trasladar todos esos principios a una nueva disciplina marcial dentro del tradicional budo, generando un arte marcial

\footnotetext{
17 Morihei Ueshiba, conocido entre sus discípulos como «O Sensei», que traducido vendría a ser el equivalente a "Gran Maestro», nació el 14 de diciembre de 1883 en la actual prefectura de Tanabe, un pequeño pueblo situado cerca de Osaka en Japón. Su padre Yoroku Ueshiba era descendiente de samuráis. Desde muy joven comenzó a practicar ejercicio físico y artes marciales, para combatir su naturaleza enfermiza (seguramente provocada por haber nacido prematuramente, según cuentan sus biógrafos), caracterizándose siempre por la búsqueda de la perfección personal. A lo largo de su vida practicó diferentes artes marciales y en diferentes escuelas (sable -Kenjutsu—, lanza —-Sojutsu—, bastón —Bojutsu—, Jujutsu, etc.). Fue una persona muy religiosa y perteneció a la secta neo-sintoísta Omoto. A primeros de los años cuarenta comenzó a utilizar el término aikido para designar el arte marcial que él mismo había elaborado (la palabra aikido se deriva de tres kanjis japoneses que significan: AI unión, armonía, coordinación-, KI —energía vital—y DO —Camino, vía, búsqueda, método de aprendizaje-). Morihei Hueshiba falleció el 26 de abril de 1969 a la edad de 86 años.
} 
nuevo denominado aikido ${ }^{18}$, en el que de forma asombrosa se produce una culminación evolutiva que lleva a concebirlo con base en unos principios que resultan ser infinitamente superiores a los que pretenden transmitir las artes marciales competitivas y el deporte en general ${ }^{19}$. Frente a estos últimos el aikido propone la introducción de las siguientes ideas:

${ }^{18}$ El aikido es un arte marcial que tiene sus raíces en el aikijutsu y que fue desarrollado, tal y como hemos indicado, por el citado maestro Morihei Ueshiba a mediados del siglo xx. Si bien el aikido como arte marcial que es, se basa en el combate entre dos oponentes, no consiste en la aplicación de una serie de técnicas agresivas cuyo objetivo último es acabar con el contrario, sino que su principal objetivo es neutralizar cualquier posible agresión reconduciendo la energía del oponente hasta anularla. Esto permite controlar las situaciones conflictivas minimizando la posibilidad de provocar daño o herir al oponente. Trata de transmitir, al que en principio es un agresor, la inutilidad de su acción, de tal manera que sólo si insiste en su violencia se verá dañado. De esta manera se aprovecha la energía del oponente reconduciéndola en su contra si no rectifica su actitud agresiva. Por ello, el aikido se caracteriza por la escasa utilización de fuerza, lo que permite que cualquiera pueda practicarlo acomodándolo a sus peculiaridades física, sexo, edad, etc. Sus técnicas permiten al aikidoka (persona que practica el aikido) ser capaz de controlar la agresividad del otro, y la propia, reconduciéndolas hacia un equilibrio de no violencia. Parte de la filosofía predicada por el aikido nace de la idea de que la mejor forma de solucionar conflictos es la búsqueda de un entendimiento mutuo, de un equilibrio donde las partes salgan beneficiadas sin imposiciones, coacción o violencia. Se trata de unas ideas que se encuentran muy arraigadas en algunas filosofías orientales como el Taoísmo que plasman esa actitud pacífica y dialogante en textos como este:

La perfección del que imparte órdenes es ser pacífico, del que combate, carecer de cólera; del que quiere vencer, no luchar; del que se sirve de los hombres, ponerse por debajo de ellos.

Sobresalir es vencer sin luchar, es convencer sin hablar, hacer acudir sin llamar, realizar a la perfección con aparente inercia...

(TAO-Te KING)

A este respecto, resultan verdaderamente llamativas las múltiples similitudes del pensamiento filosófico taoísta de la antigua China, con muchos de los postulados del liberalismo austriaco, como muy bien supo ver y analizar Murray N. Rothbard en su obra titulada Historía del Pensamiento Económico, referenciada en la bibliografía del presente trabajo.

${ }^{19}$ Más aún el deporte profesional, que además ha ido trasmitiendo, con el transcurso del tiempo, ideas altamente perniciosas como las de que sólo la victoria tiene valor $\mathrm{y}$, si no se gana, se es un fracasado, que se debe ganar a toda costa —-más aún con 
1. La superación de uno mismo, frente a la superación del adversario.

2. Respeto hacia el contrario (al que ya no se ve como tal), frente a la búsqueda de su derrota y su humillación.

3. Reconducción y neutralización de las situaciones de conflicto, frente a la confrontación directa.

4. Colaboración, por encima de la competencia despiadada.

5. Búsqueda de paz, equilibrio y enriquecimiento mutuo, frente a la idea de lucha, destrucción de la competencia y búsqueda de la obtención de beneficio particular a toda costa.

6. Respeto a los principios éticos del budo más evolucionado y que resultan compatibles con los principios anteriores (en muchos puntos coincidentes con los principios generales del derecho, los principios en los que se sustenta el liberalismo, los Tria Iuris Praecepta, y los principios de la caballería medieval - de origen eclesiástico-): Rectitud (honradez y justicia), coraje y valor, benevolencia y compasión, respeto y cortesía, honestidad y sinceridad, honor y gloria, deber y lealtad, etc., etc., etc.

Resultando todo ello, también, perfectamente compatible con nuestra cultura occidental (de base greco-romana-cristiana) a pesar de provenir de un país tan lejano como Japón, ya que son principios perfectamente válidos para cualquier sociedad humana evolucionada.

Además de los principios indicados, la práctica del aikido transmite una serie de habilidades que también son perfectamente extrapolables al liderazgo, la función empresarial (según ha sido estudiada por autores como Israel M. Kirzner y Jesús Huerta de Soto $)^{20}$ y al Management. Entre otras, dichas habilidades serían las siguientes:

\footnotetext{
la fuerte motivación económica que hay detrás de muchos de estos deportes-, lo que ha acarreado todo los problemas a los que ya nos hemos referido en líneas anteriores referentes al dopaje, los problemas psicológicos y psiquiátricos de muchos deportistas de élite, durante y después de finalizar sus carreras, así como los sobornos y las compras de partidos, etc.

${ }^{20}$ En obras como por ejemplo: Kirzner, Israel M. (1998). Competencia y Empresarialidad. Unión Editorial., Madrid, o en Huerta de Soto, J. (2002). Socialismo, Cálculo Económico y Función Empresarial. Unión Editorial, Madrid.
} 
1. Control de la acción y aumento de la capacidad de anticipación (dentro siempre de una ética defensiva).

2. Dinamismo y equilibrio.

3. Estrategias de neutralización (con tres conceptos fundamentales en base a los cuales se desarrollan las técnicas y que son: las líneas de unificación de fuerzas y las figuras de la esfera y de la espiral, como formas de canalización de la energía).

4. Flexibilidad, coordinación y preparación física.

5. Gran potenciación de la resiliencia (ya que nos enseña, no sólo a asumir con flexibilidad situaciones límite y traumáticas, sino que, además, nos enseña a sobreponernos a ellas, saliendo fortalecidos de las mismas).

6. Concentración, mokuso, meditación -mindfulness- y técnicas de respiración.

7. Aprender a caer y levantarse (en sentido físico y metafórico).

8. Respeto al compañero y camaradería (Higiene, etiqueta, etc.).

Como ya hemos mencionado, el aikido puede practicarse a cualquier edad y por cualquier persona, incluso por personas discapacitadas. No obstante, es conveniente que se comience a practicar desde joven, ya que la interiorización de las enseñanzas y los principios se hace con mayor facilidad, casi sin darse cuenta, luego sólo queda desarrollarlos lo máximo posible.

Lamentablemente, el aikido no es un arte marcial muy extendido en España, aunque hay personajes famosos que lo practican y que, incluso, han hecho campañas publicitarias basadas en él, como es el caso de la campaña para la marca de coches Jeep que hizo el torero Francisco Rivera para las televisiones españolas. Sin embargo, sí se encuentra muy extendido por otros países europeos como Francia, Alemania, Inglaterra, Rusia, etc., así como en los EEUU, donde es muy conocido tras la aparición de las primeras películas del actor y maestro de aikido, Steven Seagal. También se han introducido muchas de sus técnicas en las coreografías de escenas de lucha de películas como «El caso Burne» (y su saga), «Transporter» (y su saga), «Venganza» (y su saga), y un largo etcétera. Lamentablemente, la imagen que suelen transmitir este tipo de películas, es extremadamente violenta y agresiva - como exige la industria cinematográfica de Hollywood-, y totalmente apartada del verdadero espíritu de 
este bello arte marcial. Como suele decirse: un martillo puede servir para tallar una preciosa escultura o, mal utilizado, puede servir para matar a alguien, siendo el mismo martillo.

Llegados a este punto, lo que suele preguntarse el aikidoka es: si efectivamente el aikido tiene tanto que aportar y a tantos niveles diferentes, incluyendo evidentemente el que aquí nos ocupa del liderazgo y el Management ¿Cuál es la causa de que no se haya aplicado o consolidado de forma práctica? La respuesta en realidad puede ser muy sencilla: simple desconocimiento, ya que nadie se ha preocupado por difundir de forma adecuada sus principios y enseñanzas, por lo que se trata de un auténtico desconocido que debemos y tenemos la obligación de descubrir.

\section{$\mathrm{V}$ \\ LA APORTACIÓN DEL AIKIDO \\ EN LA FORMACIÓN DE LÍDERES, EN LAS TÉCNICAS DE MANAGEMENT, Y EN EL DESARROLLO \\ DE LA ÉTICA EMPRESARIAL Y PROFESIONAL}

Todos estos principios, habilidades y técnicas que integran el aikido son perfectamente extrapolables al mundo empresarial y al mundo de los negocios, resultando muy sencilla la elaboración de ejemplos y casos prácticos que faciliten su aplicación, pudiendo obtenerse grandes beneficios a nivel personal y colectivo, haciéndonos mejores y más equilibrados por dentro, y facilitando que las personas sean capaces de unirse para conseguir las metas que se hayan propuesto, en un continuo proceso de crecimiento y enriquecimiento mutuo continuado y estable. No obstante, y al margen de ejemplos o casos, la mejor clase práctica que podría impartirse sería observar una exhibición de lo que es el desarrollo de una clase de aikido, explicando, punto por punto, como se plasman en la realidad de una rutina práctica, todos y cada uno de los puntos analizados en el presente traba$\mathrm{jo}^{21}$.

${ }^{21}$ Una clase completa de aikido (con una duración normal de una hora o una hora y media), tendrá normalmente las siguientes partes: 


\section{El lider}

Si entendemos por líder a aquella persona a la que un grupo reconoce y acepta voluntariamente como guía, jefe u orientadora, es fácil comprender la importancia que tiene que los líderes sean personas que se guíen por unos principios éticos adecuados. Es aquí donde el aikido puede hacer una importante aportación si gracias a la enseñanza de sus principios se consigue formar líderes que guíen su actuación, y la del grupo que dirigen, orientados por los principios ya indicados de resolución de conflictos de forma pacífica, buscando el beneficio de todas las partes, reconduciendo las energías —en principio enfrentadas—, unificándolas y generando sinergias, sacando lo mejor de cada uno de los componentes del grupo que lideran, pero generando a su vez la cohesión y el compañerismo entre todos; todo ello de una forma equilibrada, pero dinámica y ágil, obteniendo resultados de forma eficaz y eficiente.

1. Previamente al inicio de la clase: es importante cuidar la higiene personal y del dojo, así como efectuar los correspondientes saludos de cortesía hacia el dojo, a la fotografía de Morihei Ueshiba, al sensei y al resto de compañeros.

2. Mokuso: breve momento de meditación.

3. Calentamiento: ejercicios respiratorios y de concentración, ejercicios de estiramiento de la columna vertebral y todas las articulaciones, ejercicios educativos específicos del aikido como tai sabaki, etc.

4. Ukemis: ejercicios para aprender a rodar y caer sin dañarse.

5. Técnicas de aikido: elegidas por el sensei para explicarlas con detalle y desarrollarlas ese día (puede haber técnicas efectuadas desde el suelo, estando de pié, de defensa contra armas, con ataques múltiples, etc.), siempre efectuadas colaborando tori y uke. El sensei siempre controlará la forma, la actitud, la corrección, etc., con que se realizan los ejercicios, ayudado si es necesario por los alumnos de mayor graduación, tratando siempre que la clase sea lo más amena y agradable para todos. A algunos profesores les gusta dedicar los últimos minutos de la clase al manejo de algunas de las armas utilizadas en aikido como el boken o el jo. Otros, sin embargo prefieren dejar algún día específico de la semana para ello.

6. Enfriamiento: ejercicios suaves de estiramiento y respiración para finalizar la clase.

7. El profesor puede aprovechar este momento para hacer algún comentario sobre el desarrollo de la clase, aclarar algún extremo o hacer algún anuncio, etc.

8. Mokuso: breve momento de meditación.

9. Saludos de cortesía al final de la clase y agradecimiento al sensei por la clase.

10. Salida ordenada del dojo y aseo personal. 
Debemos recordar que el hecho de ser líder no es bueno o malo en sí mismo, sino que depende de los medios y fines propuestos en el desarrollo de cada acción o proyecto desarrollado bajo su dirección. Es decir, el buen líder no sólo debe de ser capaz de conseguir que otros libremente le sigan y respeten, sino que, además, debe conseguir que las metas propuestas se alcancen respetando unos principios éticos básicos que todos conozcan. Esto permite, además, que la cualidad de líder sea más fácil de mantener en el tiempo y que los logros alcanzados y las metas conseguidas también lo sean a largo plazo — visión de futuro-y que beneficien a la sociedad en su conjunto — visión global—, dejando de lado la cultura del «pelotazo» y de la ganancia rápida a toda costa.

El respeto a unos principios correctos, como los que hemos indicado que forman parte de la esencia del aikido, de una forma inquebrantable es lo que transfiere carisma al líder (al maestro, sensei...) y le hace ser respetado por sus seguidores directos y por toda la colectividad. Además, el líder debe saber rodearse de los mejores seguidores (discípulos, alumnos...), y debe organizarlos adecuadamente, sabiendo además sacar lo mejor de cada uno de ellos, aprovechando sus puntos fuertes y perfeccionando los débiles.

Los seguidores y colaboradores de un líder tienen que saber, además, que es una persona honesta y que no les abandonará a mitad de camino, por lo que confían en él. Esto implica en muchas ocasiones una gran dosis de valor, lo que supone, no sólo saber enfrentarse a situaciones complicadas (que en la clase de aikido serían: el ataque o el ataque múltiple o con armas), sino también aceptar críticas y rectificar errores con rapidez, reconociéndolos públicamente si es necesario.

Finalmente, otras características o aspectos importantes que deben tenerse en cuenta en un líder, son la visión estratégica y la capacidad de adaptación que faciliten el desarrollo de las acciones adecuadas que culminen con la consecución de los objetivos propuestos. En definitiva, todos los principios, habilidades y características que venimos exponiendo del aikido. 


\section{Las técnicas de management y la dirección empresarial (colaboración -> enriquecimiento mutuo)}

La idea de Management lleva implícitas las de dirección, administración y gestión, ya que se refiere a aquella actividad encaminada a conseguir que las personas se unan y colaboren en los procesos encaminados a la consecución de fines que satisfagan necesida$\operatorname{des}^{22}$; si dichos procesos se refieren a la organización de negocios y la consecución y satisfacción de intereses económicos, nos estamos refiriendo al Management empresarial, lo que implica que existan otros ámbitos del Management no empresariales, y que se refieren a organizaciones no estrictamente económicas, sino con fines políticos, deportivos, religiosos, etc.

De esta definición se desprende con claridad la íntima relación existente entre la idea de liderazgo, anteriormente vista, y la del Management, pudiendo concluirse que un buen manager deberá reunir las cualidades que un buen líder debe tener, por lo que le son de aplicación los mismos principios que hemos comentado en el punto anterior. La única diferencia entre el Management empresarial y el resto, y la idea de líder referida a la dirección de empresas y el resto, sólo se encuentra en el tipo de análisis y resolución de casos concretos en los que tengan que aplicarse los referidos principios.

Aunque las empresas son organizaciones de tipo económico, y sabiendo que su fin es maximizar valor, también debe tenerse en cuenta que no debe abordarse su estudio desde un punto de vista estrictamente economicista, ya que existen otros tipos de necesidades y de fines (tanto internos como externos) que deben ser tenidos en cuenta, y que serán valorados internamente por los miembros de la propia organización y, externamente, por clientes, colaboradores, competidores, etc. Intentando siempre conseguir un equilibrio donde todos ganen, y en todos los aspectos, desde los estrictamente económicos hasta los que podríamos denominar más humanistas o más centrados en el propio valor de los individuos.

${ }^{22}$ En este campo de estudio, que en definitiva se refiere al desarrollo de la acción humana y de las relaciones de intercambio, resulta muy importante la aportación efectuada por L. Mises en su obra La acción humana, referenciada al final de este trabajo. 
De esta manera, lograr identificar los principios éticos que deben inspirar a la organización, con los principios éticos de los componentes de la misma; y la identificación de los fines de la organización, con los de los individuos que la integran, es función del manager. Conseguir esto es algo muy complicado, pero resulta fundamental para el buen funcionamiento y desarrollo de las empresas y de las organizaciones en general.

\section{La cultura empresarial y la ética de las organizaciones y de los individuos}

Por cultura empresarial se entiende que es el conjunto de principios, valores, etc., que caracterizan a una empresa (también puede estar referida a las organizaciones en general). Evidentemente, no tienen porqué coincidir con los de los individuos que la integran, pero no cabe duda de que la referida integración es más fácil y se beneficia si son similares o muy coincidentes.

A este respecto, los principios más generales y los valores y habilidades que el aikido puede aportar al ámbito empresarial en particular, y que hemos detallado en líneas precedentes, son más que deseables como parte integrante de cualquier cultura empresarial. Además hay otras muchas enseñanzas que ayudarían a poder alcanzar un perfecto funcionamiento interno de las empresas y a una mejor imagen de éstas en la sociedad:

a) La práctica del aikido aporta una idea de jerarquía - esencial en toda organización- básicamente formal, que se basa esencialmente en la experiencia y en los conocimientos de los practicantes, pero que, además, son unos conocimientos que en el tatami se ponen al servicio de los alumnos con grado inferior y de los aprendices. Todo cinturón negro (alumno ya iniciado en la práctica y con experiencia) debe ayudar al resto, corrigiendo con máxima paciencia y respeto, y siendo consciente de que los errores de los demás también fueron de él. Recordando en todo momento la máxima que ya hemos indicado de la filosofía taoísta que viene a decir que "La perfección del que imparte órdenes es ser pacífico; del que se sirve de los hombres, ponerse por debajo de 
ellos», con un trato siempre paciente y respetuoso. No se tiene a la información y al conocimiento como algo que confiera algún tipo de poder secreto que no debe ser compartido, sino que existe la obligación de transmitirlo de la mejor forma posible. A su vez, el más inexperto debe también tratar con respeto a los más experimentados y al resto de compañeros, y mostrar agradecimiento por los consejos y las correcciones recibidas, esforzándose al máximo por mejorar. No hay «broncas», no hay humillaciones, ni malos modos, etc., sino respeto y colaboración.

b) Los errores no se entienden como algo que deba ser castigado, sino como algo prácticamente inevitable en el proceso de aprendizaje, algo que hay que reconocer con honradez y con rapidez, para buscar una solución rápidamente, agradecer las posibles correcciones recibidas y tratar que no vuelvan a suceder. Esta actitud afecta tanto al maestro como a los alumnos (al líder o manager, como a todo el equipo que dirigen).

c) El respeto mutuo y sincero es algo que resulta fundamental. En aikido ese respeto se aprecia desde el mismo inicio de las clases con lo que se conoce como rei-gi o reglas de cortesía. El ritual de saludos (a la memoria de O Sensei, al maestro y a los compañeros), el cuidado de la higiene personal y del dojo, el mantenimiento del orden, etc., deben ser algo básico.

d) En el aikido no existe ningún tipo de discriminación, cualquier practicante, sea hombre, mujer, niño o persona mayor, es siempre bien recibido, pertenezca a la raza o religión que sea, siempre y cuando respete los principios básicos, ya mencionados, en los que se fundamenta el aikido. Expertos y neófitos practican juntos, hombres y mujeres también, ya que no hay categorías separadas ni por sexos, ni por pesos corporales, etc. Sólo hay un único objetivo: disfrutar de la práctica mejorando uno mismo y ayudando a mejorar a los demás.

f) El aikido también enseña que el trabajo debe ser sincero y honesto (por encima de objetivos particulares, número de horas trabajadas, etc.) lo que implica la utilización de medios adecuados para fines legítimos, dentro de las normas y ética establecidas.

g) La idea última sería la de realizar un trabajo serio y profesional, en un entorno agradable y de compañerismo que nos permita 
disfrutar de lo que estamos haciendo, mejorando personal y profesionalmente, como único camino para que la empresa (o la organización de que se trate) sea verdaderamente productiva, no sólo a corto plazo, sino, sobre todo a largo plazo.

Por lo que respecta a la ética empresarial o institucional, es importante destacar que aunque verdaderamente, en última instancia, son los individuos los que generan comportamiento éticos o no éticos (y que esto es verdaderamente lo fundamental ${ }^{23}$ ), no puede decirse que esté de más que en el seno de las organizaciones en general, y en las empresas en particular, se trate de buscar una guía de comportamiento en lo que se conocen como códigos de conducta o códigos éticos, que traten de mejorar la calidad ética de la organización como colectivo que persigue determinados fines propios, al margen de los de cada uno de los individuos que los forman, que, como es normal perseguirán los suyos. Evidentemente, la búsqueda de lo que podría denominarse entornos institucionales «limpios» $\mathrm{o}$ «sanos» no puede sustituir a la virtud de los individuos, pero sí que facilitará que sea mucho más sencillo y agradable realizar el trabajo asignado, produciéndose además una mayor sensación de pertenencia e identificación con la organización.

${ }^{23}$ El motivo de argumentar que lo que verdaderamente importa es la ética individual se debe a que, en realidad, una sociedad con individuos que tengan unos principios éticos adecuados y sólidos, no necesitará principios de tipo colectivo; sin embargo, esto no sucede al revés, ya que, por mucho contenido ético que se quiera dar a una organización, del tipo que sea, si los individuos que la integran no los tienen o no son los adecuados, no servirá de nada, pues son estos últimos los que en definitiva dirigen y guían la actividad organizativa y terminarán por "contaminarla». Ahora bien, es conveniente, y no está demás, que las organizaciones elaboren sus propias normas éticas de funcionamiento (interno y externo) que permita comprobar cuales son los principios que deben guiar su actividad. Al igual que en un momento dado en el derecho se generó la idea de personalidad jurídica (que en realidad es una ficción), frente a la idea de personalidad física, también cabe la posibilidad de que para las acciones desarrolladas por las personas jurídicas se quiera que deban estar guiadas por unos determinados principios éticos, aunque resulte evidente que quienes en realidad actúan, respetan o incumplen normas son los individuos que están detrás de las organizaciones y no éstas (motivo por el que, por ejemplo, se elaboró la teoría jurídica del «levantamiento del velo societario», buscando tratar de evitar posibles abusos y actuaciones de mala fe a este respecto). 
Dentro de estos códigos de conducta ética suele integrarse la denominada responsabilidad social corporativa (RSC) o responsabilidad social empresarial (RSE), que serían aquellos puntos básicos dentro de la actividad de la empresa que han sido integrados por ésta, como aporte o contribución a la mejora de la sociedad, de la economía y del medio ambiente, lo que sin duda contribuirá a largo plazo a mejorar la imagen de la empresa, favoreciendo su situación competitiva y aumentando su valor. De esta manera, el fin fundamental de toda empresa, que es obtener beneficio económico, satisfaciendo las necesidades de los individuos en el mercado, se ve reforzado gracias a su aportación social y al respeto al medio ambiente. La contribución que aquí puede aportar el aikido también es evidente, al fomentar la actividad colaborativa, la paz social, la no discriminación, etc. Además de su continua búsqueda de la integración y el equilibrio con las fuerzas del Universo y entre el ser humano y la naturaleza ${ }^{24}$.

VI

\section{CONCLUSIONES}

Al plantearnos la realización del presente trabajo nos propusimos varios objetivos distintos pero relacionados entre sí:

a) Por un lado, dejar claras las diferencias existentes entre los principios y fundamentos del aikido respecto del resto de artes marciales de contacto y de los deportes de competición. Señalando especialmente que el aikido posee todas las virtudes de estos, pero carece de sus más graves inconvenientes y contradicciones ${ }^{25}$, sien-

${ }^{24}$ El maestro M. Ueshiba decía: «El aikido no es un método para atacar y vencer al enemigo. El fin del aikido es entrar en armonía con el Universo, haciendo de nosotros un elemento integrante del mismo...».

${ }^{25}$ El deporte competitivo lanza además toda una serie de mensajes engañosos y contradictorios que todos hemos podido oír en un momento u otro, más aún los que hayan practicado deporte de cierto nivel, como por ejemplo:

1. En el deporte se debe respetar al contrario,... pero tienes que ganar a toda costa, y si es posible dándole una paliza o por el máximo de puntos o goles posible (es decir: humillándole). 
do un instrumento de formación —a todos los niveles: físico, mental y espiritual-, que por sus grandes cualidades no debe ser desperdiciado. Es más, al comprobar las conclusiones a las que hemos llegado, podría decirse que debería ser algo casi obligado para todo docente, maestro o coaching, investigar en este campo y tratar de introducir en las enseñanzas impartidas a sus discípulos, tanto la práctica del aikido — en todo lo posible-, como el aprendizaje de todo lo que de beneficioso nos pueda transmitir este arte marcial de la paz. Consideramos que las habilidades y los principios que desarrolla son, a la vez, mucho más adecuados y correctos para su aplicación en la formación de líderes, en el Management, o en la elaboración de códigos éticos, que los principios derivados de las ideas de competencia, lucha de contrarios, conquista de mercados, victoria del más fuerte, etc. Todos ellos sacados de los planteamientos de los deportes competitivos y de las artes marciales de combate. Planteamientos que en una sociedad evolucionada, justa y libre deberían de ser superados.

b) Por otro lado, y como segundo objetivo derivado del anterior, exponer cuales son esos principios y fundamentos del aikido de una forma más concreta, analizando la trascendencia que a nivel social y del mercado pueden tener si se aplican correctamente,

2. Debes respetar al compañero,... pero, por encima de eso, debes superarle para que no te quite el puesto o tú puedas quitárselo a él.

3. Lo importante no es ganar, sino participar,... pero en realidad hay que ganar cueste lo que cueste si no quieres bajar de categoría, ser eliminado, etc.

4. Hay que respetar las reglas y al árbitro,... pero son los propios entrenadores los que no dudan en insultar e increpar al arbitro, incitando a jugadores y al público.

5. Lo importante es superarse uno mismo,... pero si no superas las marcas de clasificación o las del contrario habrás fracasado y todo tu entrenamiento no valdrá para nada.

6. Etc., etc., etc.

¿Cabe mayor sarta de absurdos y contradicciones? Y si todo esto se lleva al nivel de la alta competición se acentúa mucho más, al desarrollarse en entornos muy hostiles que encierran gran agresividad y violencia, donde todo está controlado por intereses mediáticos y la obtención de grandes cantidades de dinero, de forma rápida y a costa de lo que sea, con unos niveles de presión desproporcionados, donde los errores son duramente castigados. ¿Es este el modelo que queremos para nuestros jóvenes? ¿Son estas las pautas de conducta que queremos que guíen a nuestros líderes políticos, empresariales, etc.? ¿O resultan más adecuados los principios propuestos en el presente trabajo? 
tratando de comprobar y demostrar la importancia que tiene el hecho de que se introduzcan en los programas de formación para líderes, en los cursos de Management y de formación de Directivos de empresas y organizaciones, etc., así como en la enseñanza en general. De tal manera que, aunque cuando se practica el aikido no se hace pensando en ninguna situación fuera del tatami, no cabe duda de que sus enseñanzas trascienden esa simple actividad, generando muchos frutos no buscados, pero altamente beneficiosos para el desarrollo de la vida en sociedad y para el correcto funcionamiento del mercado ${ }^{26}$, si lo que se pretende alcanzar es un crecimiento máximo de la riqueza y de la paz social de una forma sostenible, para lo cual el aikido puede contribuir al enseñarnos a:

1. Canalizar las energías negativas propias y de terceros (violencia, enfados, ataques de otros...), reconduciéndolas hasta dominarlas y transformarlas en positivas.

2. Buscar la unión de energías positivas, potenciándolas y multiplicándolas (reforzando las sinergias y los efectos feedback).

3. Alcanzar situaciones de equilibrio (en ocasiones difíciles de hallar porque el equilibrio no siempre está en el punto medio, y hay que saber buscarlo en cada situación).

4. Lograr desarrollos y crecimientos duraderos y sostenibles.

5. Potenciar trabajos en colaboración con los demás y en equipo.

6. Aumentar la capacidad creativa y de resolución de problemas.

7. No discriminación y máximo respeto hacia el otro.

8. Actuar por responsabilidad personal, y no movidos sólo por la consecución de premios y por alcanzar metas, sino disfrutando de cada momento en sí mismo.

9. Fomentar la autosuperación y la autoestima, pero comprendiendo que si ayudamos a los demás y les hacemos mejorar, también mejoramos nosotros mismos y el entorno (se trata de convertir al contrario y competidor en colega y colaborador).

${ }^{26}$ Tal y como bien ha sabido apreciar el profesor Gabriel J. Zanotti, doctor en Filosofía, Director Académico del Instituto Acton Argentina y gran aikidoka, que además insiste en el hecho de que el aikido es el arte de la paz, porque no hay en él ninguna violencia interna que esté esperando para salir, sólo hay defensa de la vida. 
10. Respeto a las normas básicas que fomentan y favorecen el trabajo y la vida en sociedad (normas éticas, morales, normas de buena conducta y cortesía, así como de higiene y orden personal y del entrono).

11. Aumentar las capacidades mentales de autocontrol, la de meditación, la concentración, así como la capacidad de resiliencia y de superación del dolor y las dificultades.

12. Mejora de innumerables aspectos físicos con la práctica continuada: flexibilidad, elasticidad, resistencia, equilibrio, velocidad, etc.

13. Facilita alcanzar la paz exterior e interior, contribuyendo a hacernos mejores personas con el consiguiente reflejo en nuestro entorno familiar, de trabajo y social. Etc., etc.

Por último, indicar que con el presente trabajo no hemos pretendido dar soluciones mágicas y definitivas, que en realidad no existen, ya que el propio aikido está siempre en proceso de evolución y en ocasiones es mal interpretado, sino que simplemente hemos querido llamar la atención sobre algunos extremos de la formación de líderes, del Management, de la ética empresarial, etc. y de la enseñanza en general, que consideramos deben tenerse en cuenta, contribuyendo con esta pequeña aportación, tratando de corregir errores del pasado e intentando mejorar nuestra sociedad con vistas al futuro y a las siguientes generaciones. Comprobando, igualmente, que esa paz, ese progreso y esa prosperidad que se lograrían aplicando los ideales expuestos en este trabajo, se integran de una forma natural y lógica con los principios y máximas defendidos por el liberalismo y por la Escuela Austríaca de Economía.

\section{REFERENCIAS BIBLIOGRÁFICAS}

Covey, S. (1997). Los 7 hábitos de la gente altamente efectiva. Barcelona. Paidós.

DruCKeR, P. (1954). The Practice of Management. Nueva York. Harper \& Row.

EDELMAN, A. J. (1994). «The implementation of a video-enhanced aikido-bases school violence prevention training program to 
reduce disruptive and assaultive behavior among severely emotionally disturbed adolescents». Fort Lauderdale, FL.Nova Southeastern University (ERIC Document Reproduction Service $\mathrm{n}^{\circ}$ EC 3040227).

FinKLER, P. (1997). Comunicar y dialogar: el arte de entender a los demás. Madrid. San Pablo, DL.

Gasalla, J. M. (2000). La nueva dirección de personas. Marco paradójico del talento directivo. Madrid. Pirámide.

Gernigon, C. y Le Bras, H. (2000). «Achievement goals in aikido and judo: a comparative study among beginner and experienced practitioners». Journal of Applied Sport Psychology, 12(2), 168179.

HAYEK, F. A. (2013). Derecho, legislación y libertad. Madrid. Unión Editorial.

- (2013). La fatal arrogancia. Madrid. Unión Editorial.

HuerTa De SOTO, J. (2002). Socialismo, Cálculo Económico y Función Empresarial. Madrid. Unión Editorial.

KING, L. A. y Williams, T. A. (1997). "Goal orientation and performance in martial arts». Journal of sport behaviour, 20(4), 397-412.

Kirzner, IsRael M.(1998). Competencia y Empresarialidad, $2^{a}$ edición. Madrid. Unión Editorial.

Martínez, C. (2006). La teoría evolutiva de las instituciones. Madrid. Unión Editorial.

Menger, C. (1997). Principios de Economía Política. Madrid. Unión Editorial.

Mises, L. (2013). La acción humana. Madrid. Unión Editorial.

MonACIS, L., y otros. (2015). «Factores motivacionales relacionados con la agresividad en las artes marciales». Revista de Psicología del Deporte. Vol 24, no 1, pp. 163-169.

N. Lussier, R. y Achur, C. (2002). Liderazgo. Teoría, aplicación, desarrollo de habilidades. Thomson Learning.

Protin, A. (1989). Aikido: un arte marcial. Barcelona. Editorial Ibis.

Ricard, C. (2008). Aikido. Práctica y sensaciones. Barcelona. Editorial Océano.

Rothbard, Murray N. (1999). Historia del Pensamiento Económico (vol. I y II). Madrid. Unión Editorial.

Sánchez, T. (2001). Aikido (El maestro y el método). Móstoles -Madrid-. Arkano Books. 
SAntos NALDA, J. (2008). Enciclopedia del aikido (cuatro tomos). Barcelona. Editorial Alas.

SAotome, M. (2000). Aikido o la armonía de la naturaleza. Barcelona. Editorial Kairós.

Sмith, Adam (2002). La riqueza de las Naciones. Madrid. Alianza Editorial.

TAmura, N. (2007). Aikido (Etiqueta y transmisión). Badalona -Barcelona-. Editorial Paidotribo.

ToHeI, K. (1966). This is aikido. Tokyo.

Tzu, S. (Edición de Samuel B. Griffith 2008). El arte de la Guerra. EVERGREEN GMBH, Köln.

UeshibA, M. (2007). Budo. (Las enseñanzas del fundador). Madrid. Dojo Ediciones.

- (2012). El arte de la paz. Madrid. Mandala Ediciones.

- (2009). Aikido paso a paso: una guía práctica. Barcelona. Editorial Kairós.

UeshiBA, K. (1963). Aikido. Tokyo.

- (2008). El espíritu del aikido. Madrid. Dojo Ediciones.

WestBROOK, A. y RATTI, O. (2001). Aikido y la esfera dinámica. Barcelona. Ediciones Obelisco. 
